\title{
Proteomic Analysis of Oxidatively Stressed Pancreatic Adenocarcinoma BxPC-3 Cells: Identification of Potential Biomarkers and Therapeutic Targets
}

\author{
Hem D Shukla ${ }^{1 *}$ and Paul Vaietiecunas ${ }^{2}$ \\ ${ }^{1}$ Department of Pharmaceutical Sciences, University of Maryland, Baltimore, USA \\ ${ }^{2}$ Department of Biology, The Johns Hopkins University, Baltimore, USA
}

\begin{abstract}
Pancreatic cancer is one of the most aggressive human malignancies and ninth leading cause of cancer death in the world. Most patients diagnosed with pancreatic cancer die within 6 months, and only $4 \%$ survive 5 years after diagnosis. Approximately one-fifth of patients with presumably 'curable' Pancreatic Ductal Adenocarcinoma (PDA) experience impending relapse and death, making surgical removal almost futile. Early diagnostic, prognostic and predictive biomarkers and better therapeutic options which could help personalize treatment regimens are desperately needed to improve the survival rate of pancreatic cancer patients. By employing BxPC-3 cell line model, high throughput comparative, quantitative and system proteomic analysis, we have been able to identify annexin-a4, annexin-a5 annexin a-11, caveolin-1, EGFR, H-RAS, Integrin-a6ß4 proteins which were significantly upregulated in oxidatively stressed BxPC-3 cells. The present investigations have shown the presence of quite robust oxidative response in BxPC-3 cells as compared to HPDE control. The high throughput proteomic and bioinformatic analysis have shown the aberrantly regulated Cav-1-Fyn-SOS-cRAF-ERK (where Cav-1 is caveolin-1) signaling pathway, reveals that oxidative stress might activate Cav-1 protein in the membrane which in turn activate the downstream proto-oncogene tyrosine-kinase Fyn and triggers MAPK/ERK pathway. The data suggest that the above stated proteins could be used as diagnostic and prognostic biomarkers and using Cav-1-Fyn-SOS-cRAF-ERK pathway better therapeutic options could be explored.
\end{abstract}

Keywords: Prognostic biomarkers; Pancreatic cancer; Pancreatic ductal adenocarcinoma

\section{Introduction}

Pancreatic cancer is one of the most aggressive human malignancies and ninth leading cause of cancer death in the world [1]. Estimated new cases and deaths from pancreatic cancer in 2013 in the United States would be 45,220 and deaths 38,460 . Most patients diagnosed with pancreatic cancer die within 6 months, and only $4 \%$ survive 5 years after diagnosis. Pancreatic cancer is characterized by colossal local invasion and early metastatic growth to the liver and regional lymph nodes [2]. At metastable stage surgical removal is the only reliable curative treatment. The incidence rate of pancreatic cancer has increased significantly in recent years [3]. It is often detected in stage III resulting in an un-resectable tumor at the time of diagnosis. However, even if pancreatic cancer is surgically resected in stage I or II, it may recur at a metastatic site. Pancreatic Ductal Adenocarcinoma is classified into resectable $(\sim 10-20 \%)$, locally advanced un-resectable $(\sim 30-40 \%)$, and metastatic ( 50\%) [3].

Because of nonspecific biomarkers for Pancreatic Ductal Adenocarcinoma (PDAC), which lack specificity and sensitivity [4], high numbers of PDAC cases are diagnosed too late in the disease process for surgical resection to be an effective option. Even among the $10-20 \%$ of PDAC cases where surgical resection is an option, most patients ultimately die of recurrent or metastatic disease [5]. Data suggest that pancreatic cancer results from the successive accumulation of gene mutations [6]. In initial stages cancer originates in the ductal epithelium and evolves from premalignant lesions to fully invasive cancer. The lesion called pancreatic intraepithelial neoplasia is the bestcharacterized histologic precursor of pancreatic cancer [7]. The recent line of investigations suggests the successive accumulation of mutations that include activation of the KRAS2 oncogene, inactivation of the tumor-suppressor genes like CDKN2A and TP53 and SMAD4 [8]. This sequence of events in pancreatic carcinogenesis is supported by studies in genetically engineered mouse models in which targeted activation of Kras2 with concomitant inactivation of Trp53 or Cdkn2A results in the development of pancreatic cancer. The recent extensive genetic analysis of 24 pancreatic cancer genomes exhibited an average of 63 genetic abnormalities per tumor, mainly point mutations. These abnormalities can be organized in 12 functional cancer-relevant pathways [9].

Early diagnosis and better treatments are desperately needed to improve the survival rate of pancreatic cancer patients. The problem of early diagnosis is further complicated by the obscure location of the pancreas, the absence of reliable symptoms and the insensitivity of biomarkers [4]. A thorough understanding of the specific cellular and molecular mechanisms of PDAC development and progression is required in order to identify early detection strategies, preventative measures, and effective interventions. Thus involving BxPC-3 cancer cell lines as a model system remains convenient for biomarker and pathway discovery using genomic and proteomic technologies. Further, cell lines recapitulate the genomic events leading to neoplastic changes. The rapidly growing field of proteo-genomics offers an opportunity

*Corresponding author: Hem D. Shukla, Department of Pharmaceutical Sciences, University of Maryland, Baltimore MD21201, USA, Tel: 410-746-4794 Fax: 410-746-4250; E-mail: Hshukla@rx.umaryland.edu

Received October 04, 2013; Accepted October 30, 2013; Published November 04, 2013

Citation: Shukla HD, Vaietiecunas P (2013) Proteomic Analysis of Oxidatively Stressed Pancreatic Adenocarcinoma BxPC-3 Cells: Identification of Potential Biomarkers and Therapeutic Targets. J Mol Biomark Diagn 4: 149. doi:10.4172/2155-9929.1000149

Copyright: (c) 2013 Shukla HD, et al. This is an open-access article distributed under the terms of the Creative Commons Attribution License, which permits unrestricted use, distribution, and reproduction in any medium, provided the original author and source are credited 
to identify the cellular and molecular risk factors associated with pancreatic cancer. Herein, we report the proteomic analysis of oxidative stress response in BxPC- 3 cells and its comparison to HPDE control cells. An attempt has also been made to perform comparative and quantitative proteomic investigations and identification of promising biomarkers in cancer cells. In addition, the high through put proteomic analysis and systems biology approach was also adapted to identify the aberrantly regulated signaling pathway in Pancreatic Ductal Adenocarcinoma (PDAC).

\section{Material and Methods}

\section{Cell culture, oxidative stress and Cell lysate preparation}

Pancreatic adenocarcinoma BxPC3 cancer cell lines were cultured in Dulbecco's Modified Eagle's Medium (DMEM) with 10\% FBS (Gibco). Immortal Human Nontumorigenic Pancreatic Duct Epithelial Cells (HPDE6) was cultured in Clontech KGM-2 karotinocyte media. All cell lines were grown at $37^{\circ} \mathrm{C}$ with $5 \% \mathrm{CO}_{2}$. Cell lines are tested monthly for Mycoplasma contamination using Venor GeM Mycoplasma Detection Kit, PCR based from Sigma. Cells were allowed to grow at a cell density of $1.5 \times 104$ cells per well of a 96 -well plate at $37^{\circ} \mathrm{C}$ in $5 \% \mathrm{CO}_{2}$ in their recommended growth media containing $10 \% \mathrm{FCS}, 2 \mathrm{mmol} / \mathrm{L}$ glutamine (Invitrogen). EC50 concentration of $\mathrm{H}_{2} \mathrm{O}_{2} 150 \mu \mathrm{mol} / \mathrm{L}$ was mixed with cells and treated for 2 hours. The medium was then removed and cells were washed and cultured in growth medium for an additional $22 \mathrm{~h}$ without hydrogen peroxide. Cells were harvested and processed for cell lysate preparation.

BxPC-3 and HPDE cell lines were suspended in $150 \mu \mathrm{l}$ of $50 \mathrm{mM}$ Tris- $\mathrm{HCl}, 0.2 \%$ SDS, $\mathrm{pH}$ 8.0. Tubes were homogenized and chilled on ice for $30 \mathrm{~min}$ and sonicated for $10 \mathrm{~min}, 30 \mathrm{sec}$ pulse and $30 \mathrm{sec}$ off. The total cell lysate was centrifuged at $10,000 \mathrm{rpm}$ for $10 \mathrm{~min}$ at $4^{\circ} \mathrm{C}$, and supernatant was transferred into clean tubes. Supernatant was transferred into respective tubes as cell lysate fraction. The final sell lysate was used for protein estimation by BCA method (Pierce).

\section{SDS-PAGE analysis}

Samples with $100 \mu \mathrm{g}$ per lane of proteins from BxPC and HPDE were subjected to SDS-Polyacrylamide gel electrophoresis (SDS-PAGE) under reducing conditions on commercial small gradient (NOVEX, 4-20\%). The samples were electrophoresed for $2 \mathrm{hrs}$ at 125 volts at $4^{\circ} \mathrm{C}$, and the gel was rinsed 3 times with Milli Q water and stained with Simple blue stain for O/N. Subsequently, the gels were destained with milli Q water and bands were excised and processed. Then each gel was carefully laid on a glass plate and horizontal slices were cut with new razor blades for each sample and cleaning the razor between slices. Each gel slice was cut into $\sim 1 \mathrm{~mm}$ cubes and was processed for in-geldigestion.

\section{Sample preparation for proteomic analysis}

The coomassie stained gel pieces from BxPC-3 (pancreatic cancer) and HPDE samples (control) were carefully sliced with sterile blade and chopped into $0.5 \mathrm{~mm}$ square pieces using Fisher brand Microscope slides each time. Gel pieces were carefully transferred into $1.5 \mathrm{ml}$ sterile appendorf tubes and were destained in 5\% acetonitrile and $75 \%$ of 25 $\mathrm{mM}$ Ammonium bi-carbonate $(\mathrm{ABC})$. Further, the gel pieces were completely dehydrated in $400 \mu \mathrm{l}$ of $100 \%$ acetonitrile for $10 \mathrm{~min}$. and discarded the acetonitrile. In the last step after removing acetonitrile the gel pieces were completely dried for Reduction and Alkylation. The reduction was performed in appendorf tubes by adding $10 \mathrm{mM}$ of DTT and tubes were vortexed well and incubated at $55^{\circ} \mathrm{C}$ for $45 \mathrm{~min}$ under shaking condition. Subsequently, the gel pieces were alkylated in 55 $\mathrm{mM}$ of freshly prepared iodoacetamide and incubated in dark for 30 $\mathrm{min}$ at RT. Then the gel pieces were incubated in $400 \mathrm{ul}$ of $5 \mathrm{mM} \mathrm{ABC}$ for $30 \mathrm{~min}$ at room temperature and solution was discarded. In final step gel pieces were dehydrated in $400 \mu \mathrm{l}$ of acetonitrile for $10 \mathrm{~min}$ and gel pieces were completely dried before trypsin digestion.

Trypsin digestion was performed in trypsin digestion buffer and trypsin to protein ratio was adjusted 1:20 ratio. The tubes were vortexed and incubated on ice for $45 \mathrm{~min}$, and enough digestion buffer was added to cover the gel pieces and tubes were incubated at $37^{\circ} \mathrm{C}$ for overnight. Following day tubes were centrifuge at $6000 \mathrm{rpm}$ for $5 \mathrm{~min}$. and the liquid containing peptides was collected into a clean tubes. The resulting tryptic peptides were initially extracted two times with $50 \mu \mathrm{l}$ of $50 \%$ acetonitrile, $45 \%$ HPLC grade distilled water and $0.5 \%$ formic acid. During extraction tubes were incubated at RT for $30 \mathrm{~min}$ and in the last step peptides were eluted one time with $50 \mu$ of $100 \%$ acetonitrile. The extracted peptides were vacuum dried in Savant Speedvac centrifuge under low temperature settings. $10 \mu \mathrm{l}$ of sample buffer was added to dissolve peptides and injected to LTQ-ETD-MS for further analysis.

\section{LC-MS/MS analysis}

Samples $(10 \mu \mathrm{l})$ were injected into online Michrom Paradigm MS4 LC using an autosampler (CTC-Autosampler, CTC Analytics) with a flow rate of $20 \mathrm{ul} / \mathrm{min}$. The digested peptides were trapped into microPrecolumn C18 trap (Dionex Corporation) and separated using RP C18 $75 \mathrm{uM} 10 \mathrm{~cm}$ analytical column (New Objective). The separated peptides were sprayed into a LTQ linear ion trap mass spectrometer (ThermoElectron) for LC-MS-MS analysis as described by Johanssen et al. [10]. During LC, a linear gradient from $98 \%$ mobile phase A ( $2 \%$ acetonitrile, $0.1 \%$ formic acid, $98 \% \mathrm{H}_{2} \mathrm{O}$ ) to $90 \%$ mobile phase $\mathrm{B}$ ( $2 \% \mathrm{H}_{2} \mathrm{O}, 0.1 \%$ formic acid, $97.9 \%$ acetonitrile) was used for $110 \mathrm{~min}$ followed by $98 \%$ mobile phase A for $10 \mathrm{~min}$. The peptides were detected in positive ion mode in LTQ linear ion trap in a data-dependent mode in which the seven most abundant ions detected in an initial survey scan were selected for MS-MS analysis. Each scan cycle consisted of one full scan mass spectrum (m/z 400-1800) collected in profile mode followed by six MS-MS events in centroid mode. The quantitative analysis of raw files generated from young and old thymocytes were performed using MS Decyder software (GE HealthCare, NJ), and raw files were searched against Human NCBInr database to get identification using BioWorks 3.3.1 Sp1 (Thermo Scientific) .

\section{Quantitative analysis}

The raw data files were analyzed by Spectrum Mill proteomics software (Rev A03.02.060, Agilent Technologies, Santa Clara, CA). The extracted files were searched against the human non-redundant International Protein Index (IPI) human sequence database (http:// www.ebi.ac.uk) using trypsin as the protease with one missed cleavage and fixed modification of carbamidomethylation and variable modifications of oxidized methionine and N-terminal glutamine conversion to pyroglutamic acid in the search. Proteins with minimum two validated peptides with 25 score or more were considered significant. To compare identified proteins in oxidatively stressed BxPC- 3 and HPDE control runs, the number of spectra and the Total Ion Intensity (TII) were used as indicators of fold changes in protein quantity. Because these were semi quantitative metrics, we added total intensities for each protein in all three runs for BxPC-3 and normal HPDE control separately and divided them to calculate the protein ratio. If the protein level was elevated greater than or equal to 1.5 or less than or equal to 0.67 were considered significant. Cellular locations of 
Citation: Shukla HD, Vaietiecunas P (2013) Proteomic Analysis of Oxidatively Stressed Pancreatic Adenocarcinoma BxPC-3 Cells: Identification of Potential Biomarkers and Therapeutic Targets. J Mol Biomark Diagn 4: 149. doi:10.4172/2155-9929.1000149

the proteins were determined by using protein center program version 2.01 (PROXEON, Denmark).

\section{Result}

\section{Comparative proteomic analysis of BxPC-3 and HPDE cells}

The proteomic analysis of BxPC-3 pancreatic cell lines was performed by preparation of cell lysates from both BxPC-3 and HPDE control cells. The equal amount of the proteins were resolved on to $4-12 \%$ SDS-PAGE, and subsequently processed for destaining. The gel bands were sliced and processed for in-gel-digestion and LC-MS/MS analysis. In order to gain higher confidence level in present analysis, we selected the protein which had minimum two peptides. In the present report we have been able to identify 1860 proteins in BxPC-3 and 644 protein in HPDE and The Protein Center analysis has shown that 1715 proteins were common in both HPDE and BxPC-3 as shown in (Figure 1A). However, the data analysis of BxPC-3 and HPDE control based on single peptide have shown the presence of 3756 proteins common in both HPDE and BxPC-3, and 1330 proteins were unique in HPDE, and 3471 in BxPC-3 cells. The pathway analysis indicated that there were 68 pathways were found in HPDE and 91 were found in BxPC-

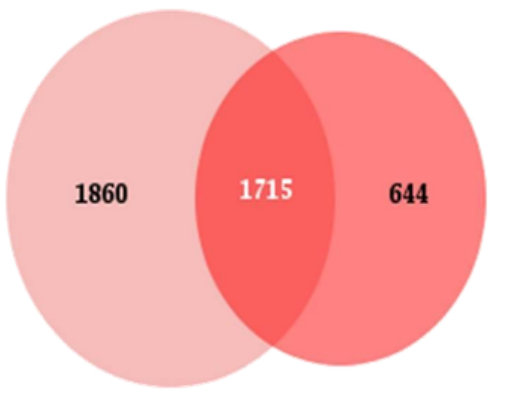

BXT

HPT

Figure 1A: Comparative analysis of the proteins identified in oxidatively stressed BxPC-3 cells and HPDE control.

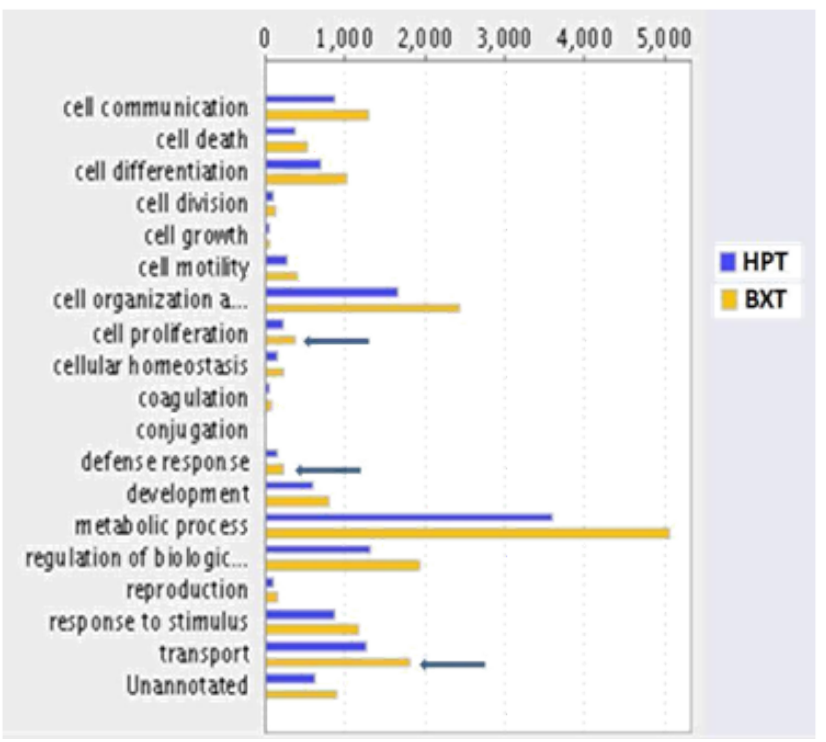

Figure 1B: The GO analysis of biological function category in BxPC-3 cells and HPDE control.
3 pancreatic cancer cell lines. The comparative analysis of oxidatively stressed BxPC-3 and HPDE control cell under GO biological function category has shown significant difference in cell proliferation, defense response and transport category (Figure 1B). Interestingly under cell proliferation category annexin-A1, annexin-a4, annexin-a5 annexin a-11, caveolin-1, EGFR, H-RAS, Integrin- $\alpha 6 \beta 4$, KRAS, SET and stress induced phosphor-protein were quite prominent in BxPC-3 cells as compared to HPDE control. The previous reports have confirm the presence some of these gene products [11-13]. The comparative analyses of host defense category have shown the presence of peroxiredoxin-2, peroxiredoxin-4, superoxide mutase (Mn-SOD) mitochondrial, superoxide disputase-2, catalase, glutathione peroxidase-1 in BxPC3 cells. The corollary finding also suggest the presence of strong oxidative stress response in BxPC-3 cancer cells which impart an edge to cancer cells for stress adaptation [14-17]. In pancreatic ductal adenocarcinomas, the majority of the exocrine pancreatic tumors are thought to develop in a multistep process, involving a series of specific genetic, epigenetic and environmental factors which are involved in the genesis and development of this disease [18]. The pathogenesis of this disease is through accumulation of genetic and molecular changes, resulting in defects in the growth, adhesion and integration of the pancreas. Recently, the comprehensive genetic analysis of 24 pancreatic cancers has shown 63 genetic alterations, and majority of which are point mutations [9]. Interestingly, these alterations have been shown to affect 12 signaling pathways leading to pancreatic tumorigenesis [19]. The recent reports also suggest that nine genes of DNA repair pathways have been found to be mutated in pancreatic cancer [9].

The presence of strong oxidative stress response in cancer cells allow cancer cells to quickly adapt to oxidative stress and make these cell drug resistant [20,21]. The present data suggest the aberrant regulation of redox homeostasis and stress adaptation in cancer cells (Figure 1B). The evidence suggests that, compared with their normal counterparts, many types of cancer cell have increased level of reactive oxygen species [17]. Under persistent oxidative stress, many cancer cells become well-adapted to such stress and develop an enhanced, endogenous antioxidant capacity, which makes the malignant cells resistant to exogenous stress [16]. As a consequence cancer cells that survive intrinsic oxidative stress may have activated adaptive mechanisms, which switch on ROS-scavenging systems to cope with the stress. Recent evidence suggests that such adaptation contributes to malignant transformation, metastasis and resistance to anticancer drugs [22]. This approach may have might be a potential to develop therapy for pancreatic cancer [23].

\section{Quantitative proteomic analysis of BxPC-3 cells and identification of biomarkers}

The analysis with Agilent's Spectrum Mill software as described in the methods and the autovalidation feature of this software is unique and has been successfully used by several investigators [23-25]. Most of the proteins were found to be present in all three runs of both BxPC-3 and HPDE control samples, while some of the gene products were found only in BxPC-3 cells or control samples. There were also significant differences in the levels of certain proteins present in cancer cells as compare to normal control samples. Several criteria were used to determine which proteins displayed a significant increase or decrease in their level in cancer cells in comparison to normal control. Protein entries with only one peptide match were considered insignificant and were discarded. After the spectrum mill analysis there were 10 proteins observed in BxPC- 3 cells which exhibited more than 3.5 fold increase as compared to normal control. Proteins which were exclusively present 
Citation: Shukla HD, Vaietiecunas P (2013) Proteomic Analysis of Oxidatively Stressed Pancreatic Adenocarcinoma BxPC-3 Cells: Identification of Potential Biomarkers and Therapeutic Targets. J Mol Biomark Diagn 4: 149. doi:10.4172/2155-9929.1000149

Page 4 of 5

\begin{tabular}{|c|c|c|}
\hline Protein name & Location & Regulation \\
\hline Caveolin-1 & Membrane & Up \\
\hline EGFR & Membrane & Up \\
\hline C-H-RAS & Membrane & Up \\
\hline Integrin $\beta 1 R$ & Membrane & Up \\
\hline Galectin-1 & Membrane & Up \\
\hline SET nuclear & Nucleus & Up \\
\hline Oncogene & & \\
\hline Annexin-a2 & Membrane & Up \\
\hline Integrin $\alpha 6 \beta 4$ & Membrane & Up \\
\hline R-HOC & cytoplasmic & Up \\
\hline Tryptophan-5 & Cytoplasmic & Up \\
\hline Mono-oxygenase & & \\
\hline Activation protein & & \\
\hline
\end{tabular}

Table 1: Quantitative analysis of gene products up-regulated in BxPC-3 cells by spectral counting.

\begin{tabular}{|c|c|c|c|c|}
\hline Accn no. & Gene & BxPC-3 cells & HPDE Control & Description \\
\hline Q8HXP7 & SOD2 & Present & Absent & $\begin{array}{l}\text { Superoxide dismutase } \\
\text { [Mn], mitochondrial }\end{array}$ \\
\hline IPI00027350.3 & PRDX2 & Present & Absent & Peroxiredoxin-2 \\
\hline IPI00465436.4 & CAT & Present & Absent & Catalase \\
\hline 4467837 & GPX1 & Present & Absent & Glutathione peroxidase \\
\hline IPI00024919.3 & PRDX3 & Present & Absent & $\begin{array}{l}\text { Thioredoxin-dependent } \\
\text { peroxide reductase, } \\
\text { mitochondrial }\end{array}$ \\
\hline
\end{tabular}

Table 2: Oxidative stress induced proteins in BxPC-3 cells.

in BxPC-3 cells and several fold upregulated were considered potential biomarkers candidates [14]. The quantitative proteomic analysis have shown the presence of annexin-a2, annexin-a5, CRAS, Galectin-1, SET, integrin $\alpha 6 \beta 4$, integrin $\beta-1 \mathrm{R}$ which were significantly upregulated in BxPC-3 (Table 1). The earlier reports have also shown the upregulation of annexin-a2, integrinal alpha6 beta4 and EGFR in pancreatic cancer cell as potential biomarkers for pancreatic cancer [26-28].

\section{Oxidative stress response of BxPC-3 cells and oxidative therapy}

Under oxidative stress conditions pancreatic cancer cells induced and synthesized SOD2-mitochondrial, peroxiredoxin-2 and 4, and glutathione peroxidase, (Table 2). The present data suggest the aberrant regulation of redox homeostasis and stress adaptation in cancer cells $[20,29]$. Mounting evidence suggests that, compared with their normal counterparts, many types of cancer cell have increased level of reactive oxygen species. Under persistent oxidative stress, many cancer cells become well adapted to such stress and develop an enhanced, endogenous antioxidant capacity, which makes the malignant cells resistant to exogenous stress. Recent evidence suggests that such adaptation contributes to malignant transformation, metastasis, and resistance to anticancer drugs.

Currently, there is an anticancer strategy known as "oxidative therapy", which consists of inducing high ROS steady state levels in tumor cells. Causing the generation of high levels of ROS in solid tumors and inhibiting the antioxidant system of tumor cells [20]. High levels of ROS induce apoptosis in a wide variety of tumor cells activating the caspase cascade.

Increased oxidative stress may exert harmful effects including damage to DNA and cell membranes, leading to carcinogenesis and tumor progression and overproduction of Reactive Oxygen and Nitrogen Species (RONS) and have been implicated in the pathogenesis of pancreatitis, both in its acute and chronic form, as well as in the pathogenesis of Pancreatic Cancer (PC) [30]. The increased oxidative stress associated with the induction of pancreatic cancer correlates well with other studies in various model systems which demonstrate that ROS can initiate and promote carcinogenesis. It has been proposed that increased oxidative stress and Reactive Oxygen Species (ROS) generation, caused by overexpression of membrane non-mitochondrial Nicotinamide Adenine Dinucleotide Phosphate (NADPH)-oxidase, is connected with pancreatic (patho) biology such as cell growth regulation and apoptosis, acinar cell inflammation, fibrosis, and disturbed islet microcirculation [31].

\section{Development of therapeutic approaches to treat pancreatic cancer}

The main reason for the failure of current conventional therapy to cure pancreatic cancer and the major cause for cancer-related mortality in general is the ability of malignant cells to detach from the primary tumor site and develop metastasis in different regions of the same organ and in distant organs. In addition, pancreatic cancer is a highly chemoresistant malignancy. It has been suggested that cellular stress might be responsible in phosphorylating Cav-1, which activate downstream signaling and may play important role in Cav-1 function (Figure 2). Thus, Cav-1 may be useful target to improve conventional therapies, including radiotherapy for pancreatic cancer. Another key pathway of integrin signaling is focal adhesion kinase, which diversely interacts with cytoskeletal proteins, which are important in the formation of focal adhesions, and play role in activation of integrin signaling involving MAPK kinase, c-jun N-terminal kinases (JNK) pathway. In particular, integrin signaling pathway is the subject of intense research scrutiny which has potential for developing pharmacologic inhibitors for the treatment of cancer. Interestingly, the inhibition of integrin heterodimer $\alpha \mathrm{V} \beta 6$ resulted in a significant reduction in cell proliferation and invasion, and it could be used as therapeutic target against pancreatic cancer adenocarcinoma [32]. The experimental evidence suggest that caveolin- 1 expression was up regulated in $\mathrm{BxPC}$ 3 cells and the interactions of Cav-1 with 1-integrin or FAK affect radiation survival and proliferation of pancreatic carcinoma cells. The data suggest that Cav-1 is critical to these processes and could be used for prognostic purposes [32].

In the present work three promising biomarkers have been identified

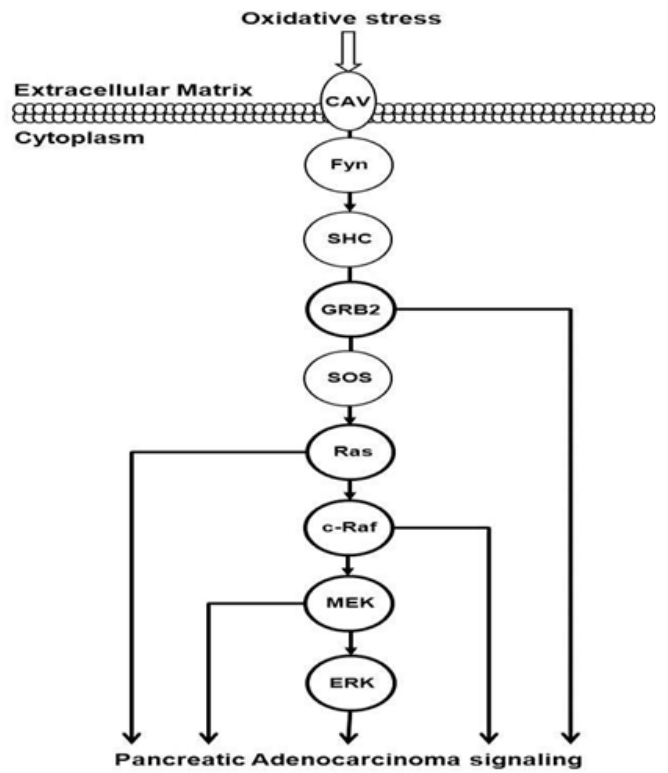

Figure 2: Oxidative stress induced signaling in pancreatic cancer (BxPC-3), which abnormally regulates caveolin-1 (Cav-1) on the membrane, which in turn interact with Fyn kinase and triggers downstream PDAC signaling. 
Citation: Shukla HD, Vaietiecunas P (2013) Proteomic Analysis of Oxidatively Stressed Pancreatic Adenocarcinoma BxPC-3 Cells: Identification of Potential Biomarkers and Therapeutic Targets. J Mol Biomark Diagn 4: 149. doi:10.4172/2155-9929.1000149

which could be used for early diagnosis. Further, the high throughput proteomic analysis has allowed identification of Cav-1-Fyn-SOS-cRAFERK pathway which seems to have potential therapeutic significance.

\section{Acknowledgement}

This work was supported by a research grant from Department of Biology, Johns Hopkins University, Baltimore, MD 21201. Authors thanks Dr. Cotter's lab for running mass spec experiments.

\section{References}

1. Hezel AF, Kimmelman AC, Stanger BZ, Bardeesy N, Depinho RA (2006) Genetics and biology of pancreatic ductal adenocarcinoma. Genes Dev 20 1218-1249.

2. Hartwig W, Hackert T, Hinz U, Gluth A, Bergmann F, et al. (2011) Pancreatic cancer surgery in the new millennium: better prediction of outcome. Ann Surg 254: 311-319.

3. Ying H, Kimmelman AC, Lyssiotis CA, Hua S, Chu GC, et al. (2012) Oncogenic Kras maintains pancreatic tumors through regulation of anabolic glucose metabolism. Cell 149: 656-670.

4. Grønborg M, Kristiansen TZ, Iwahori A, Chang R, Reddy R, et al. (2006) Biomarker discovery from pancreatic cancer secretome using a differentia proteomic approach. Mol Cell Proteomics 5: 157-171.

5. Real FX, Malats N, Lesca G, Porta M, Chopin S, et al. (2002) Family history of cancer and germline BRCA2 mutations in sporadic exocrine pancreatic cancer. Gut 50: 653-657.

6. Vogelstein B, Kinzler KW (2004) Cancer genes and the pathways they control. Nat Med 10: 789-99.

7. Hruban $\mathrm{RH}$, Maitra A, Goggins M (2008) Update on pancreatic intraepithelia neoplasia. Int J Clin Exp Pathol 1: 306-316.

8. Feldmann G, Beaty R, Hruban RH, Maitra A (2007) Molecular genetics of pancreatic intraepithelial neoplasia. J Hepatobiliary Pancreat Surg 14: 224-232.

9. Jones S, Zhang X, Parsons DW, Lin JC, Leary RJ, et al. (2008) Core signaling pathways in human pancreatic cancers revealed by global genomic analyses. Science 321: 1801-1806.

10. Johansson C, Samskog J, Sundström L, Wadensten H, Björkesten L, et al. (2006) Differential expression analysis of Escherichia coli proteins using a novel software for relative quantitation of LC-MS/MS data. Proteomics 6: 44754485 .

11. Farina A, Dumonceau JM, Frossard JL, Hadengue A, Hochstrasser DF, et al. (2009) Proteomic analysis of human bile from malignant biliary stenosis induced by pancreatic cancer. J Proteome Res 8: 159-169.

12. Chen R, Pan S, Brentnall TA, Aebersold R (2005) Proteomic profiling of pancreatic cancer for biomarker discovery. Mol Cell Proteomics 4: 523-533.

13. Cecconi D, Donadelli M, Dalla Pozza E, Rinalducci S, Zolla L, et al. (2009) Synergistic effect of trichostatin A and 5-aza-2'-deoxycytidine on growth inhibition of pancreatic endocrine tumour cell lines: a proteomic study. Proteomics 9: 1952-1966

14. Shukla HD, Vaitiekunas $P$ (2011) Proteomic analysis of integrin signaling pathway in pancreatic carcinoma cell lines (BxPC-3) and targets for therapeutic intervention. 7th EDRN Workshop, NCI P6.

15. Shukla HD, Vaitiekunas $P$, Cotter RJ (2012) Advances in membrane proteomics and cancer biomarker discovery: current status and future perspective. Proteomics 12: 3085-3104.

16. Sullivan R, Graham $\mathrm{CH}$ (2008) Chemosensitization of cancer by nitric oxide. Curr Pharm Des 14: 1113-1123.

17. Egler RA, Fernandes E, Rothermund K, Sereika S, de Souza-Pinto N, et al. (2005) Regulation of reactive oxygen species, DNA damage, and c-Myc function by peroxiredoxin 1 . Oncogene $24: 8038-8050$.
18. Jones S, Hruban RH, Kamiyama M, Borges M, Zhang X, et al. (2009) Exomic sequencing identifies PALB2 as a pancreatic cancer susceptibility gene. Science 324: 217.

19. lacobuzio-Donahue CA (2012) Genetic evolution of pancreatic cancer: lessons learnt from the pancreatic cancer genome sequencing project. Gut 61: 10851094.

20. Trachootham D, Alexandre J, Huang P (2009) Targeting cancer cells by ROSmediated mechanisms: a radical therapeutic approach? Nat Rev Drug Discov 8: 579-591.

21. Pani G, Galeotti T, Chiarugi $P$ (2010) Metastasis: cancer cell's escape from oxidative stress. Cancer Metastasis Rev 29: 351-378.

22. Chen El, Hewel J, Krueger JS, Tiraby C, Weber MR, et al. (2007) Adaptation of energy metabolism in breast cancer brain metastases. Cancer Res 67: 1472 1486.

23. Fandiño AS, Rais I, Vollmer M, Elgass H, Schägger $H$, et al. (2005) LCnanospray-MS/MS analysis of hydrophobic proteins from membrane protein complexes isolated by blue-native electrophoresis. J Mass Spectrom 40: 12231231.

24. Hörth P, Miller CA, Preckel T, Wenz C (2006) Efficient fractionation and improved protein identification by peptide OFFGEL electrophoresis. Mol Cell Proteomics 5: 1968-1974.

25. Stevens AL, Wishnok JS, Chai DH, Grodzinsky AJ, Tannenbaum SR (2008) A sodium dodecyl sulfate-polyacrylamide gel electrophoresis-liquid chromatography tandem mass spectrometry analysis of bovine cartilage tissue response to mechanical compression injury and the inflammatory cytokines tumor necrosis factor alpha and interleukin-1beta. Arthritis Rheum 58: 489-500.

26. Pan S, Chen R, Reimel BA, Crispin DA, Mirzaei H, et al. (2009) Quantitative proteomics investigation of pancreatic intraepithelial neoplasia. Electrophoresis 30: 1132-1144.

27. Zhang W, Zhao P, Xu XL, Cai L, Song ZS, et al. (2013) Annexin A2 promotes the migration and invasion of human hepatocellular carcinoma cells in vitro by regulating the shedding of CD147-harboring microvesicles from tumor cells. PLoS One 8: e67268.

28. Boeck S, Jung A, Laubender RP, Neumann J, Egg R, et al (2013) EGFR pathway biomarkers in erlotinib-treated patients with advanced pancreatic cancer: translational results from the randomised, crossover phase 3 trial AIOPK0104. Br J Cancer 108: 469-476.

29. Freitas M, Baldeiras I, Proença T, Alves V, Mota-Pinto A, et al. (2012) Oxidative stress adaptation in aggressive prostate cancer may be counteracted by the reduction of glutathione reductase. FEBS Open Bio 2: 119-128.

30. Momi N, Kaur S, Krishn SR, Batra SK (2012) Discovering the route from inflammation to pancreatic cancer. Minerva Gastroenterol Dietol 58: 283-297.

31. Sarsour EH, Kumar MG, Chaudhuri L, Kalen AL, Goswami PC (2009) Redox control of the cell cycle in health and disease. Antioxid Redox Signal 11: 29853011.

32. Liu L, Xu HX, Wang WQ, Wu CT, Chen T, et al. (2013) Cavin-1 is essential for the tumor-promoting effect of caveolin-1 and enhances its prognostic potency in pancreatic cancer. Oncogene. 